\section{Cahiers de littérature orale}

$83 \mid 2018$

Geneviève Calame-Griaule

\title{
Au plus près et au-delà des mots, dans les creux de la parole. Actualité de Geneviève Calame-Griaule
}

A Quest for Hidden Treasures: Speech from Within and Without

\section{Frank Alvarez-Pereyre}

\section{OpenEdition}

Journals

Édition électronique

URL : https://journals.openedition.org/clo/4599

DOI : $10.4000 /$ clo.4599

ISSN : 2266-1816

Éditeur

INALCO

Édition imprimée

Date de publication : 21 juin 2018

Pagination : 21-34

ISBN : 9782858313143

ISSN : 0396-891X

Référence électronique

Frank Alvarez-Pereyre, « Au plus près et au-delà des mots, dans les creux de la parole. Actualité de Geneviève Calame-Griaule », Cahiers de littérature orale [En ligne], 83 | 2018, mis en ligne le 20 juin 2019, consulté le 01 juillet 2021. URL : http://journals.openedition.org/clo/4599; DOl : https://doi.org/ $10.4000 /$ clo.4599

\section{(@) $(1) \Theta$}

Cahiers de littérature orale est mis à disposition selon les termes de la Licence Creative Commons Attribution - Pas d'Utilisation Commerciale 4.0 International. 


\section{Au plus près et au-delà des mots, dans les creux de la parole. Actualité de Geneviève Calame-Griaule}

Frank Alvarez-Pereyre

UMR 7206 du CNRS, du MNHN et de l'université Paris VI

Les écrits, les recherches et les enseignements de Geneviève Calame-Griaule ont porté haut les ambitions de l'ethnolinguistique. Militante infatigable autant que posée, elle a bataillé pour faire évoluer des paysages intellectuels qu'elle considérait comme réducteurs. Elle et ceux de ses collègues qui défendaient la même cause s'avéraient constituer une minorité. Il leur fut pourtant donné d'accomplir une œuvre ample et durable, dans des institutions qui entérinèrent leurs efforts de plusieurs manières.

On aurait du mal aujourd'hui à reconnaître une telle effervescence autour des défis qu'ils avaient identifiés. Disons plus justement qu'à l'époque des pionniers ont succédé des temps où l'on laboure certes les champs qu'ils ont défrichés, mais dans un relatif émiettement individuel, alors que les disciplines frontières, qui avaient fini par avoir pignon sur rue, connaissent une éclipse durable dans les établissements de recherche et d'enseignement. Il n'en reste pas moins que les graines plantées par les pionniers, et leurs propres moissons, fécondent des travaux qui dénotent un enracinement durable des hypothèses initiales, autant qu'un travail polymorphe et innovant sur les concepts et les méthodes qui ont été mis en avant des années 1960 aux années 1990.

Trois facteurs justifient que l'on puisse tenter d'évaluer aujourd'hui les contributions propres de G. Calame-Griaule à plusieurs champs des humanités.

D'une part, le succès durable du paradigme cognitiviste interpelle profondément ceux des travaux qui s'attachent à la diversité des cultures 
entendue comme une problématique anthropologique incontournable. Pourtant, et parallèlement, l'intérêt pour les contextes d'interaction, les stratégies d'agents et la performance ne cesse de croître et de s'affermir.

D'autre part, une interrogation frontale ${ }^{1}$ relative à la saga indo-européenne sollicite les sciences humaines et sociales dans leurs tréfonds. Une vision centralisatrice, fortement idéologique, se trouve ici clairement mise en cause, l'ethnolinguistique se voyant attribuer dans le même temps un statut théorique privilégié, à côté de la sociolinguistique et de la dialectologie.

Enfin, s'il est vrai que l'interdisciplinarité garde une faveur de principe dans les instances de recherche et d'enseignement, une telle faveur s'avère être superficielle dans ses effets. Plus essentiellement, l'interdisciplinarité reste interpellée par des difficultés intrinsèques qui mobilisent peu les esprits. Or de telles difficultés concernent l'appréhension méthodique de certains des caractères les plus centraux et les plus complexes de l'activité humaine. Ainsi particulièrement de l'action rituelle, entendue comme un ballet réglé de constituants à l'hétérogénéité maximale. Une importante multiplication de niveaux stratégiques intervient dans ce cadre, qui interroge de façon frontale la notion de norme, pour en repousser la compréhension. Ainsi, encore, de l'activité symbolique, qui emmène dans ses filets des composantes qui défient sans cesse le travail pourtant incontournable des spécialistes de disciplines bien identifiées. Car une telle activité cultive en continu les relations entre les plans de l'implicite et de l'explicite, de l'individu et du collectif, de l'arbitraire et de la motivation, de la diachronie et de la synchronie.

Dans une telle situation, relire et écouter G. Calame-Griaule - y compris en veillant à la situer parmi ses collègues du moment - semble faire sens, au regard des problématiques et des tensions que les éléments de contexte indiqués ci-dessus portent en eux. On procèdera en trois temps.

Il s'agit d'abord de dégager les significations que porte en elle l'inscription de la parole au centre de l'édifice théorique et pratique que G. Calame-Griaule a patiemment établi.

Il convient ensuite de comprendre la portée du choix tout particulier - et non exclusif - marqué par cet auteur pour les contes, parmi les productions qui relèvent de l'oralité.

En dernier lieu, il nous faut tenter d'évaluer les raisons et les implications des propos très concrets que G. Calame-Griaule a progressivement élaborés avec d'autres autour de l'ethnolinguistique.

1. Cf. Demoule, 2014. 


\section{L'élection de la parole}

Parmi les arguments qui fédèrent ceux qui, dans la deuxième moitié du $\mathrm{Xx}^{\mathrm{e}}$ siècle, se réclament de l'une ou l'autre tendance de l'ethnolinguistique, de la linguistique anthropologique ou de l'anthropologie linguistique, l'ethnographie de la parole occupe une place de premier rang ${ }^{2}$. Les motivations s'ancrent, à cette période et selon les auteurs, dans la conviction que la linguistique du $\mathrm{Xx}^{\mathrm{e}}$ siècle a par trop délaissé le versant plus individuel et mouvant de l'activité langagière, que les interactions verbales observées en contexte représentent une voie d'accès privilégiée vers les constructions et dynamiques sociales, que l'oralité constitue un univers dense et foisonnant, dont on peine à mesurer la complexité intrinsèque et les effets.

Dans ce contexte, G. Calame-Griaule occupe une place singulière. Tout en ne reniant pas, dans le fond, une linguistique qui vise à rendre compte des langues sur le versant structural et systémique, elle va investir résolument l'univers de la parole. Globalement, c'est spécifiquement au nom de « la relation de la société à la parole » qu'elle définit l'ethnolinguistique ${ }^{3}$. Une telle relation doit être entendue « au sens d'actualisation de la langue dans la communication ${ }^{4} \gg$. La communication est donc le lieu spécifique où l'entité langue prend vie et c'est, de façon incontournable et déterminante, au sein de la société que s'opère une telle incarnation de la langue. La parole est la manifestation tangible, au sein d'une société précise, d'une telle incarnation. Elle en est la condition. Le chercheur se doit alors d'être attentif à l'ensemble des déterminants potentiels qui peuvent entrer en jeu dans ce cadre. Il doit être capable de rendre compte des nombreuses déterminations effectives qui s'actualisent à travers l'acte de parole, qui le justifient et qui en façonnent les effets.

C'est tout particulièrement à propos de la littérature orale que G. Calame-Griaule va se faire plus précise. Elle détaillera (1970) plusieurs ensembles de facteurs qui déterminent concrètement l'acte de communication autour des répertoires de textes : les conditions sociales de la production orale, les fonctions de la littérature orale, les agents de la transmission, les traits et les procédés stylistiques. Elle reprendra ensuite les dimensions qui viennent d'être nommées pour définir de façon plus poussée les caractères fondamentaux

2. Cf. Bornand \& Leguy, 2013, p. 73-99.

3. Calame-Griaule, 1977, p. 16 et 1991, p. 631.

4. Ibid. 
de l'acte de parole auquel correspond la profération de la littérature orale en situation ${ }^{5}$.

La singularité de cet auteur tient à deux facteurs, qui sont en réalité deux facettes d'une même pièce. Les propositions de G. Calame-Griaule visaient fondamentalement à dépasser le divorce qui s'épaississait entre une langue conçue comme une entité en soi et la vie d'une telle langue au jour le jour, dans ses manifestations les plus variées. Pour ce faire, il fallait mettre en lumière l'ensemble des facteurs et des vecteurs fonctionnels mobilisés - aussi variés soient-ils -, démontrer qu'à tout moment la symphonie de ces facteurs et vecteurs implique des choix culturels significatifs, dont il revient au chercheur de révéler la nature et la pertinence pour la société considérée. Cela implique quant aux méthodes de pouvoir mobiliser rigoureusement - voire de façonner de toute pièce - des savoir-faire hétérogènes au nom des cohérences internes plus ou moins stables qui travaillent au sein d'une société donnée et qui ne se donnent pas à voir d'emblée. Au fond, il fallait, et il faut toujours, prendre la mesure de la forte complexité de l'acte de parole. Il fallait, et il faut toujours, ne pas oublier que pour chaque constituant, paramètre ou registre, les caractères formels et fonctionnels de ceux-ci se doivent d'être identifiés pour leur part propre autant qu'au nom des manières dont ils prennent part ensemble dans les configurations mouvantes et multiples des actes de parole, toujours situés et motivés.

Ce que l'on vient de rappeler parle du chercheur et des choix scientifiques qui se présentent devant lui. Sur ce versant, G. Calame-Griaule s'est faite l'avocate d'un continuum conceptuel et méthodologique, qui garde toute sa pertinence heuristique. Tout autant, elle s'est investie sur un autre versant, sans quitter sa problématique de fond. Elle a en effet poussé très loin l'hypothèse selon laquelle, en matière de parole, l'impératif du continuum scientifique pourrait aussi être appelé par l'hypothèse d'une contiguïté pensée au plan culturel ; une contiguité certes enfouie, mais bien opératoire. C'est le sens que l'on pourrait donner au travail majeur qui a abouti à l'ouvrage Ethnologie et langage (1965), dont le sous-titre est La parole chez les Dogon.

Le travail relatif à la parole chez les Dogon expose résolument comment s'opère, sur le registre de la parole et à travers elle spécifiquement, un principe généralisé de correspondances entre les dimensions structurantes et les constituants de l'univers, entre les manifestations attestées de cet univers dans lequel l'homme est partie prenante, qu'il déchiffre et sur lequel il agit. Autrement dit, l'extraordinaire multiplicité des ancrages et des caractères qui

5. Cf. Calame-Griaule, 1977, p. 23-24. 
définissent, déterminent et marquent l'humain dans sa définition propre et dans sa foisonnante relation au monde, tout cela entreait dans une pensée ordonnée, dans un double principe de contiguïé et de transposition de registre à registre. Un tel édifice - qui atteste de la complexité du monde et d'une volonté d'en penser les articulations internes au-delà de toutes les hétérogénéités irréductibles - se révèlerait spécifiquement par l'intermédiaire de la parole. Elle seule, en tant qu'elle est cet acte spécifique, de cette nature particulière, pourrait constituer le pivot central - car non unique - d'un tel principe de contiguité et de transposition, elle seule pourrait le manifester avec une telle force.

Il ne s'agirait pas ici d'un édifice idéal. Il s'agirait d'une construction sur le registre des représentations, mais des représentations qui sont élaborées à propos et au nom de ce à quoi engagent tout à la fois la part individuelle et l'engagement social de tous. À partir de là, on comprendrait combien l'ancrage social de la parole est le creuset où peuvent se jouer toutes les partitions possibles de l'entente et de la discorde, de la bonne mesure et de la contestation, des protocoles pédagogiques où se jouent les voilements et les dévoilements par où se construit l'être social, toutes les modalités normatives et leurs transgressions : que les protagonistes aient ou non clairement conscience du fait que leurs individualités sont en réalité comptables du groupe à l'intérieur duquel ils s'inscrivent peu ou prou. Sur ce registre des représentations, et mieux que sur tout autre, seraient inventoriées, seraient mises en scène et inlassablement articulées, les dimensions du social, les constituants du social, mais aussi le caractère irrémédiablement hétérogène des facteurs constitutifs de l'humain et encore la question brûlante des cohérences relatives que les humains tissent entre eux.

En tenant compte maintenant des engagements de G. Calame-Griaule aussi bien en matière de continuité sur le registre descriptif et analytique qu'au titre des contiguittés travaillées au sein même des cultures, on proposera qu'élire la parole dans ces termes et à cette place, c'est se permettre de respecter et d'articuler les paroles individuelles et les théories culturelles de la parole. C'est pouvoir transcender les clivages entre systèmes et structures d'une part, performance, interaction et contexte d'autre part. C'est encore rendre justice à la prise de parole, considérée comme cet acte immédiat ou premier où se dessinent inévitablement des fins dernières, qui deviennent à leur tour matière à négociation. 


\section{La faveur pour les contes}

Parmi les objets qu'elle a elle-même assignés à l'ethnolinguistique, G. Calame-Griaule a privilégié le domaine de la littérature orale et, dans ce domaine, elle a porté un intérêt tout particulier aux contes. C'est, de son propre aveu, une expérience de terrain qui l'aura conduite à cette préférence. Dans son introduction générale au recueil de textes qu'elle a intitulé Des cauris au marchéb, elle relate comment elle a découvert que les textes peu élaborés qui lui étaient racontés durant ses premières missions de terrain se transformeront avec le temps en des récits plus longs et bien plus complexes. Réalisant qu'elle est la destinataire d'une forme d'initiation, G. Calame-Griaule va transformer l'événement singulier en hypothèse de travail.

Un deuxième facteur va venir soutenir cette hypothèse, pour en faire le ferment d'une préoccupation majeure. Le comparatisme intra-africain mais aussi la fréquentation de l'ouvrage d'Antti Aarne et Stith Thompson (1964) vont la convaincre que les contes populaires sont une école irremplaçable, que les sociétés érigent pour leurs besoins propres. Une telle école est elle-même à entendre en plusieurs sens, ce que G. Calame-Griaule n'aura de cesse d'expliciter.

Il s'agit d'une structure d'enseignement, pour les nouveaux venus qui seront les adultes de demain, qu'il faut former au sein de leur propre société, de leur propre environnement : apprentissage du monde naturel, apprentissage des travaux et des rites, apprentissage des manières de faire société. Il s'agit également d'un lieu où une société donnée se mesure à elle-même, à ses besoins, à ses difficultés, à son histoire passée, aux défis du présent. Elle se parle à haute voix et à voix basse tout à la fois, elle pèse et évalue, construit et défait. Enfin, les mêmes contes seraient encore le creuset où se voient abordées des préoccupations fondamentales que toutes les sociétés partagent : des préoccupations qu'elles travaillent toutefois de façon indirecte et relativement voilée, dans un ballet incessant entre motifs réalistes, d'un abord immédiat, et motifs plus délicats, plus compacts, d'un abord bien moins direct.

Ce sont ces préoccupations fondamentales que G. Calame-Griaule va s'efforcer de mettre en lumière. Ce sont les chemins pris pour en traiter qu'elle va se donner pour objectif d'identifier. Pour ce faire, elle n'aura de cesse de rester au plus près des textes recueillis et de leur contexte spécifique, tout en réfléchissant aux outils et aux démarches qui lui permettront de se situer au plus près des voies

6. Calame-Griaule, 1987 , p. 7-8. 
que les textes empruntent eux-mêmes pour être ce qu'ils ont pour vocation d'être. Cela, tant du point de vue littéraire que du point de vue de leur performance.

C'est là qu'intervient une nouvelle décision, à propos de laquelle elle s'est expliquée très clairement ${ }^{7}$. Pour G. Calame-Griaule, il n'y a pas de voie unique en matière de méthode, il n'y a pas non plus de modèle unique. Un tel parti pris est à la mesure du défi que constituent les raisons d'être sociales et culturelles des textes. Si ces textes remplissent de façon si systématique des fonctions variées et complémentaires, si leur cahier des charges est à ce point élaboré et vital, si leur facture et les manipulations dont ils font l'objet sont si complexes, alors il faut humblement se mettre à la hauteur de ce par quoi ils interpellent.

L'inventaire des outils et des démarches s'établit ainsi. L'ethnographie de terrain s'impose pour ce qui touche au contexte culturel et au fonctionnement social de la littérature orale. L'attention portée à la « pensée symbolique africaine ${ }^{8} \gg$ se justifie dans la mesure où il faut pouvoir « prendre en compte les interprétations inconscientes des usagers eux-mêmes et leur vision du monde ${ }^{9} \gg$. De son côté, le structuralisme offre « la méthode qui permet de mettre en évidence les systèmes d'oppositions inconscientes qui régissent cette vision $\mathrm{du}$ monde traditionnelle et la logique symbolique de ces oppositions ${ }^{10} \gg$. Le passage par la psychanalyse est présenté comme incontournable pour « découvrir le sens caché des contes $\gg$, mais le savoir psychanalytique ne peut être appliqué de façon mécanique. Il faut le mettre en regard de la « connaissance ethnologique du contexte ${ }^{11} \gg$. La dette envers les travaux de Vladimir Propp en matière de structure narrative est clairement affichée, autant que la productivité qu'il faut reconnaître à la méthode comparatiste, toujours du côté des structures narratives et quant aux motifs symboliques.

À lire attentivement les propos de G. Calame-Griaule, à suivre patiemment les études de textes qu'elle a multipliées, il s'avère bien qu'il y a fondamentalement une attention qui est portée aux constructions du sens. Et si la variété des méthodes s'impose, c'est que les constructions de sens mettent en œuvre, simultanément, des modalités variées, différents processus, au sein d'un ample mouvement de mises en équivalence, de transpositions, de transformations.

7. Calame-Griaule, 1975 , p. 5-7 et 1987, p. 11-14.

8. Calame-Griaule, 1987, p. 12.

9. Ibid.

10. Ibid.

11. Ibid. 
Car les processus en matière de sens, s'ils relèvent d'opérations mentales, $s$ 'incarnent dans des images, dans des objets, dans des protagonistes, dans du faire tangible, que la langue va exposer à sa manière propre. C'est là que s'imposent encore d'autres méthodes. Il faut alors regarder du côté de la linguistique, de la stylistique, de la poétique, mais aussi se rendre compte que la performance passe également par un ensemble de canaux dont certains sont totalement ancrés dans l'ethnographie, quand d'autres relèvent en particulier d'une sémiologie des gestes.

Tous ces outils, toutes ces méthodes sont seconds, non pas secondaires. Ils sont tous impératifs, et tous relatifs. Ils viennent nécessairement, dans leur limite propre, à l'appui des interrogations fondamentales suivantes : comment, avec les outils de ma profession, puis-je accéder à quelque chose qui relève de pertinences qui me sont a priori étrangères, et que je me dois pourtant de restituer. Comment pouvoir rendre compte de cette insistance que mettent les sociétés dans l'élaboration à ce point minutieuse et a priori opaque de quelque chose qui doit faire sens et qui ne peut le faire que de manière détournée ? Comment approcher, puis restituer cette monumentale imbrication des plans de pertinence, qui passe par une non moins monumentale imbrication des vecteurs concrets de la signification?

On a dit à quel point l'expérience de terrain aura été essentielle dans ce qui a déterminé ses choix durables quant aux objets et ses décisions majeures quant aux méthodes. On a vu que le même auteur a validé cette expérience de terrain en se rendant compte à quel point les genres de la littérature orale étaient répandus de par le monde, et ce pour des raisons essentielles. Pour G. Calame-Griaule, les contes se seront avérés constituer des fabriques du sens à grande échelle, en même temps que des pièces incontournables du jeu social et de son devenir, dans des lieux toujours situés, dont il faut respecter au plus haut point la spécificité.

Il semble bien que se réalise là, d'une manière toute singulière, la jonction de trois plans irréductibles et pourtant totalement imbriqués : celui des opérations symboliques les plus récurrentes et les plus centrales dans la construction des individus et des sociétés ; celui des opérateurs, variés mais déterminants, par lesquels passent l'encodage et le décodage des fils multiples qu'appelle une telle construction des individus et des sociétés ; celui des ancrages les plus localisés à travers lesquels les sociétés prennent en charge leurs problématiques propres, pour les construire et les négocier au jour le jour au double plan individuel et collectif. 


\section{L'ethnolinguistique : un éclectisme bien tempéré}

G. Calame-Griaule n'a eu de cesse d'orienter les recherches relatives aux sociétés et aux cultures vers des conceptions et des pratiques décloisonnées. Un tel projet impliquait, premièrement, de rendre les scientifiques conscients de la nécessité intrinsèque des disciplines constituées, mais aussi de leurs limites objectives face à des objets toujours plus complexes que ce que chacune d'elles peut en dire. Deuxièmement, il convenait de forger des outils et des démarches qui permettent de travailler au plus près des catégories internes aux sociétés et cultures étudiées, pour mettre ces catégories en lumière dans leurs contenus et dans leurs fonctions, mais aussi pour en établir les usages et le devenir.

G. Calame-Griaule n'a pas été isolée dans cette entreprise. Elle a travaillé de concert ou en parallèle avec des collègues largement acquis aux mêmes exigences. Il est frappant, dans ce contexte, de relever quelques similitudes fortes. À lire les travaux de G. Calame-Griaule, de Jacqueline M. C. Thomas, de Maurice Houis, de Dell Hymes, mais aussi de toute une pléiade d'auteurs ayant travaillé dans leur voisinage proche, on notera que leurs contributions se répartissent sur trois registres. Il y a d'une part ce qui relève des définitions de l'ethnolinguistique, de ce qui la justifie, de ses grandes perspectives et projets. Il y a par ailleurs ce qui relève de la définition des objets pour l'ethnolinguistique, de ses thématiques et domaines d'investigation. Il y a enfin ce qui relève des méthodes.

Admettons, à ce premier niveau, que les propos de tel ou tel, et tout particulièrement de G. Calame-Griaule, sont marqués au sceau d'une stabilité et d'un éclectisme relatifs. Elle est constante dans l'énoncé des grandes thématiques qui lui semblent tout particulièrement relever de l'ethnolinguistique : langue et vision du monde, littérature orale, ethnothéories du langage, ethnographie de la parole. L'exposé de ces thèmes varie toutefois dans les détails, d'une fois sur l'autre. Lorsqu'elle explicite les différences qu'elle perçoit entre l'ethnolinguistique et la sociolinguistique, elle admet que les définitions respectives qu'elle en donne sont loin de poser des frontières étanches entre elles. Et elle ajoute à la panoplie de ses préoccupations le domaine et les pratiques des dialectologues.

On avait vu précédemment avec quelle insistance G. Calame-Griaule justifiait et assumait l'éclectisme des méthodes quand il s'agissait d'étudier la littérature orale. En matière d'ethnolinguistique, il n'y a pas eu de dogmatisme ni d'enfermement conceptuel ou pratique. Ce sont là les symptômes non pas d'une carence coupable, mais les marques d'une clairvoyance particulièrement réaliste et productive. Celle-ci porte sur les modes d'ajustement qu'il convient d'établir entre les protocoles scientifiques d'une part, et les protocoles vernaculaires 
d'autre part. Les premiers relèvent d'une philosophie de la connaissance. Les autres relèvent d'une philosophie de l'action. Et, sur la question de la responsabilité, les unes et les autres n'ont pas les mêmes cahiers des charges. Certes, G. Calame-Griaule n'a pas raisonné dans ces termes. Mais si l'on veut apprécier la nature de son héritage aujourd'hui, en regardant ce qu'elle a livré au prisme de ce que l'on appellera une épistémologie pratique dans le temps long, alors force est de constater qu'il y a, de sa part, une contribution franche à de tels débats de fond. Le scientifique doit bien se hisser à la hauteur des défis que constitue l'extrême attention que mettent les sociétés à leur construction et à leur devenir, tant au plan individuel qu'au plan collectif. À cet effet, il n'y a pas de recette universelle, il n'y a pas de modèle unique.

Le socle commun aux auteurs qui se sont réclamé de l'ethnolinguistique aura été une défiance raisonnée vis-à-vis des tentations de pureté qui avaient très largement droit de cité en linguistique et en ethnologie. De telles tentations poussaient tout à la fois au cloisonnement des disciplines, à la décomposition des objets, à leur émiettement indéfini, à une perte du sens des cohérences relatives, multiples et transitoires, qui ne cessent d'animer les sociétés et les cultures. À l'inverse, les argumentaires respectifs puis les cheminements de ceux qui se définissaient - et se définissent encore - comme ethnolinguistes, deviennent particulièrement importants quand on réalise qu' ils ouvrent des voies complémentaires et particulièrement pertinentes en matière de compréhension des sociétés et des cultures étudiées. Dans leur ouvrage intitulé Anthropologie des pratiques langagières (2013), Sandra Bornand et Cécile Leguy veillent à établir un inventaire méthodique des singularités relatives et des apports respectifs des auteurs concernés : qu'il s'agisse de définitions, d'objets ou de méthodes, précisément. Disons-le nettement : la variété des contributions complémentaires révèle tout ce qu'occultait la préférence pour les étanchéités entre disciplines, ou pour une conception complètement désincarnée des objets scientifiques. Tentons ici un bref inventaire.

L'attention méthodique portée aux champs et aux voies de l'oralité, à ses architectures, à ses dynamiques et à ses effets, à sa plasticité propre, tout cela a conduit à réévaluer à nouveaux frais les oppositions par trop figées entre écriture et oralité. Avec des effets profonds dans des domaines aussi variés que l'histoire du continent africain ou des populations d'Océanie, la compréhension des formes littéraires, voire des formes musicales qui prévalaient au Moyen-Âge, l'ethnologie des pratiques rituelles autant que de la culture matérielle, par exemple.

La transformation significative des protocoles mis en œuvre pour la description des langues à tradition orale a ouvert des perspectives insoupçonnées. 
Le nombre des monographies a connu une augmentation sans précédent, tous continents confondus, et cela a ouvert des perspectives insoupçonnées dans le domaine de la typologie linguistique.

Des réorientations profondes sont intervenues au sein même des travaux relatifs aux savoirs et aux savoir-faire, aux catégories et catégorisations endogènes. Ce qui a rendu possible cela, c'est une explicitation de la singulière centralité du langage, autant que le déploiement méthodique d'une interdisciplinarité pratique, pensée et enseignée en tant que telle.

L'habitude a été prise de diversifier les objets successifs de la recherche, de manière à explorer les ramifications multiples que connaissent une expression langagière donnée, un motif narratif, un objet matériel, les éléments d'un rituel, au nom des échos constitutifs qui se font entendre d'un registre à un autre de l'activité humaine.

Le pari fait sur la parole incarnée a permis de voir à quel point celle-ci est une voie d'accès irremplaçable vers les représentations symboliques mais aussi le creuset incontournable où se font et se défont les stratégies d'acteurs, à l'importance primordiale.

C'est plus généralement une transformation des modèles de compréhension des sociétés humaines qui a été rendue possible, sur quatre plans au moins : celui de leur écologie propre, celui de leurs modes de structuration, celui de leur historicité, celui de leurs dynamiques internes.

Si chacun des ethnolinguistes de l'époque a tracé une voie originale, c'est au nom du fait que, dès que l'on accepte d'être à ce point à l'écoute des sociétés et de l'immense travail qui y est déployé, dès que l'on entre sérieusement dans une interrogation sur les outils et les méthodes, alors c'est un chantier monumental qui s'ouvre. Dans un tel contexte, l'hypothèse d'une voie unique en matière d'appréhension des données s'avère être d'un bien piètre rendement. Car elle postulerait une essentialité de principe, à propos des manières dont une société donnée fait société.

Certes, G. Calame-Griaule, comme d'autres, n'a pas parcouru tous les champs qu'elle a élus à son programme. Elle n'a pas toujours poussé jusqu'aux confins d'un domaine ou d'une question donnés, par ailleurs explorés avec intérêt et réussite. Si elle n'a pas généreusement exploré, en particulier, ce qui allait devenir les thématiques de la performance et de l'agentivité, elle en a campé les contours, elle en a établi la pertinence de principe et elle a forgé quelques outils particulièrement précieux, qui étaient alors inexistants en la matière. Ceci étant, elle aura fondamentalement posé un mode de comportement global fait de minutie, d'un intérêt renouvelé pour les pertinences propres aux sociétés 
étudiées, pour les paroles qui, de l'individu à la collectivité, s'emboitent, se nourrissent réciproquement ou s'affrontent.

Il convient de garder ceci à l'esprit, si tant est que les démons qui habitaient la recherche scientifique et qui ont poussé à l'éclosion de l'ethnolinguistique, de l'anthropologie linguistique ou de la linguistique anthropologique n'ont pas été des démons passagers. Ils persistent tout en pouvant varier dans leurs contenus et dans leurs modes d'existence. Et personne n'est à l'abri d'une rencontre plus ou moins durable avec eux.

À bien des égards et fort heureusement il s'avère que la génération des fondateurs de l'ethnolinguistique a su susciter des travaux qui ont notablement élargi le champ des problématiques que ces fondateurs avaient ouvert. Cette même génération a conduit à des approfondissements particulièrement féconds sur bien des registres qu'ils avaient identifiés. Ce n'est donc pas seulement leur mémoire qui reste vivante à travers leurs élèves ou ceux qui se reconnaissent en eux. Ce qui reste vivant, plus généralement, c'est une manière d'interroger l'humain dans son histoire, dans ses projets et dans ses réalisations, au nom d'une précaution d'humilité pratique, face à des catégories et à des comportements toujours exotiques, dont les rationalités internes restent un défi durable.

\section{Bibliographie}

Aarne Antti \& Stith Thompson, 1964, The Types of the Folktale, Suomalainen tiedeakatemia, Helsinki, 588 p.

Bornand Sandra \& Leguy Cécile, 2013, Anthropologie des pratiques langagières, Armand Colin (coll. Collection U. Sciences humaines \& sociales), Paris, $205 \mathrm{p}$.

Calame-Griaule Geneviève, 1965, Ethnologie et langage. La parole chez les Dogon, Gallimard, Paris, 589 p.

Calame-Griaule Geneviève, 1970, « Pour une étude ethnolinguistique des littératures orales africaines $\gg$ in POTTIER Bernard (dir.), Langages, $\mathrm{n}^{\circ} 18$, p. 22-47, DOI : 10.3406/lgge.1970.2026.

Calame-Griaule Geneviève, 1975, « Introduction » in CalameGriaule Geneviève (dir.), Permanence et métamorphoses du conte populaire : 
Au PLUS PRÈS ET AU-DELÀ DES MOTS, DANS LES CREUX DE LA PAROLE. Actualité de Geneviève Calame-Griaule

Frank Alvarez-PEREyre

la mère traîtresse et le tueur de dragons, Publications orientalistes de France, Paris, p. 5-15.

Calame-Griaule Geneviève, 1977, « Introduction : pourquoi l'ethnolinguistique ? » in Calame-Griaule Geneviève (dir.), Langage et cultures africaines : essais d'ethnolinguistique, F. Maspero, Paris, p. 11-28.

Calame-Griaule Geneviève, 1987, Des Cauris au marché : essais sur des contes africains, Société des africanistes, Paris, 293 p.

Calame-Griaule Geneviève, 1992, « Ethnologie et sciences de langage » in Pottier Bernard (dir.), Les sciences du langage en France au $\mathrm{xx}^{e}$ siècle, $2^{\mathrm{e}}$ édition, Peeters, Paris, p. 626-671.

Demoule Jean-Paul, 2014, Mais ou sont passés les Indo-Européens ? Le mythe d'origine de l'Occident, Éditions du Seuil, Paris, 741 p.

Résumé : en mettant la parole au centre de ses préoccupations, Geneviève Calame-Griaule a voulu articuler paroles individuelles et théories culturelles sur la parole, pour mieux transcender les clivages entre système et structure d'une part, performance, interaction et contexte d'autre part.

Son attention pour les contes lui a permis d'opérer la jonction entre le plan des opérations symboliques les plus centrales dans la construction des individus et des sociétés et le plan des opérateurs qui permettent concrètement l'encodage et le décodage des multiples fils qu'appelle une telle construction des individus et des sociétés.

Ses propos et propositions en matière d'ethnolinguistique sont empreints d'un éclectisme raisonné. Ils se déploient sous la forme de développements théoriques mais aussi au travers d'élaborations méthodologiques minutieuses et innovantes.

Ces trois points caractérisent de façon centrale ce que fut l'activité scientifique de Geneviève Calame-Griaule de son vivant. Ils marquent tout autant sa durable actualité.

Mots-clefs : ethnolinguistique, langue, parole, littérature orale, oralité, interdisciplinarité, épistémologie, comparatisme. 


\section{A Quest for Hidden Treasures: Speech from Within and Without}

Abstract: Geneviève Calame-Griaule has been constantly dealing with speech and the verbal production, searching for cultural patterns and also for the individual impact onto it. Her favour for the folk tales can be understood as an interest for the manners a given society demonstrates its desire and capacity to teach its own value on the long run. She has been instrumental as a fervent scholar who would favor any attempt to bridge linguistics and ethnology, be it on the conceptual and theoretical level, be it on the methodological register. These traits do speak for the continuying impact of Geneviève Calame-Griaule's achievments.

Keywords: ethnolinguistics, language, speech, oral literature, orality, interdisciplinarity, epistemology, comparatism.

\section{Note sur l'auteur}

Frank Alvarez-Pereyre est directeur de recherche émérite au CNRS. Il consacre une partie importante de ses recherches et ses enseignements aux théories et méthodes de l'interdisciplinarité. 